\title{
Improved A-P Iterative Algorithm in Spline Subspaces ${ }^{\star}$
}

\author{
Jun Xian ${ }^{1}$, Shi-Ping $\mathrm{Luo}^{2}$, and Wei $\operatorname{Lin}^{1}$ \\ 1 Department of Mathematics, Sun Yat-sen University, \\ Guangzhou, 510275, China, xianjun11@sohu.com \\ 2 Department of Mathematics, South China Normal University, \\ Guangzhou, 510631, China, stslw@zsu.edu.cn
}

\begin{abstract}
In this paper, we improve A-P iterative algorithm, and use the algorithm to implement the reconstruction from weighted samples, and obtain explicit convergence rate of the algorithm in spline subspaces.
\end{abstract}

\section{Introduction}

For a bandlimited signal of finite energy, it is completely described by the famous classical Shannon sampling theorem. This classical theorem has broad application in signal processing and communication theory and has been generalized to many other forms. However, in many real applications sampling points are not always regularly. It is well-known that in the sampling and reconstruction problem for non-bandlimited spaces, signal is often assumed to belong to a shiftinvariant spaces $[1,2,4,5,7,8,9,10]$. As the special shift-invariant spaces, spline subspaces yield many advantages in their generation and numerical treatment so that there are many practical applications for signal or image processing $[1,2$, $3,9]$.

For practical application and computation of reconstruction, Goh et al., show practical reconstruction algorithm of bandlimited signals from irregular samples in [11], Aldroubi et al., present a A-P iterative algorithm in [5]. In this paper, we improve the A-P iterative algorithm in spline subspaces. The improved algorithm occupies better convergence than the old one.

\section{Improved A-P Iterative Algorithm in $V_{N}$}

Aldroubi presented A-P iterative algorithm in [5]. In this section, we will improve the algorithm. The improved algorithm occupies faster convergence. We will discuss the cases of non-weighted samples and weighted samples, respectively.

We define some symbols. $V_{N}=\left\{\sum_{k \in Z} c_{k} \varphi_{N}(\cdot-k):\left\{c_{k}\right\} \in \ell^{2}\right\}$ is spline subspace generated by $\varphi_{N}=\chi_{[0,1]} * \cdots * \chi_{[0,1]}$ ( $\mathrm{N}$ convolutions), $N \geq 1$.

\footnotetext{
* This work is supported in part by the China-NSF, the Guangdong-NSF and the Foundation of Sun Yat-sen University Advanced Research Centre.
} 
Let $\chi_{j, 1}(x)=\chi_{\left[x_{j}, \frac{x_{j}+x_{j+1}}{2}\right)}(x), \chi_{j, 2}(x)=\chi_{\left[\frac{x_{j}+x_{j+1}}{2}, x_{j+1}\right)}(x), \forall x_{j} \in X(X$ is sampling set),

$$
W\left(L^{p}\right)=\left\{f \in L^{p}:\|f\|_{W\left(L^{p}\right)}=\left(\sum_{k \in Z^{d}} \operatorname{esssup}_{x \in[0,1]^{d}}|f(x+k)|^{p}\right)^{(1 / p)}<\infty\right\}
$$

if $1 \leq p<\infty$,

$$
W\left(L^{\infty}\right)=\left\{f \in L^{\infty}:\|f\|_{W\left(L_{v}^{p}\right)}=\sup _{k \in Z^{d}} \operatorname{esssup}_{x \in[0,1]^{d}}|f(x+k)|<\infty\right\}
$$

if $p=\infty$. Let oscillation $\operatorname{osc}_{\delta}(f)(x)=\sup _{|y| \leq \delta}|f(x+y)-f(x)|$.

We show some lemmas that will be used in the proof of Theorem 2.1 , 2.2, and 2.3.

Lemma 2.1 ${ }^{[5]}$ If $\varphi$ is continuous and has compact support, then for anyf $\in V^{p}(\varphi)=\left\{\sum_{k \in \mathbb{Z}} c_{k} \varphi(\cdot-k):\left(c_{k}\right) \in \ell^{p}\right\}$ the conclusions (i)-(ii) hold:

$i .\|f\|_{L^{p}} \approx\|c\|_{\ell^{p}} \approx\|f\|_{W\left(L^{p}\right)}$,

ii. $V^{p}(\varphi) \subset W_{0}\left(L^{p}\right) \subset W_{0}\left(L^{q}\right) \subset W\left(L^{q}\right) \subset L^{q}(\mathbb{R})(1 \leq p \leq q \leq \infty)$.

Lemma 2.2 If $f \in V_{N}$, then for any $0<\delta<1$ we have $\left\|\operatorname{osc}_{\delta}(f)\right\|_{L^{2}}^{2} \leq$ $(3 N \delta)^{2} \sum_{k \in \mathbb{Z}}\left|c_{k}\right|^{2}$

Proof. We have the following equalities and inequalities:

$$
\begin{gathered}
\left\|o s c_{\delta}(f)\right\|_{2}^{2}=\int_{-\infty}^{\infty}\left|\sup _{|y| \leq \delta}\right| \sum_{n \in \mathbb{Z}} c_{n}\left(\varphi_{N}(x+y-n)-\varphi_{N}(x-n)\right) \|^{2} d x \\
\leq \int_{-\infty}^{\infty}\left(\sum_{n \in \mathbb{Z}}\left|c_{n}\right| \sup _{|y| \leq \delta}\left|\varphi_{N}(x+y-n)-\varphi_{N}(x-n)\right|\right)^{2} d x \\
=\int_{0}^{1} \sum_{k \in \mathbb{Z}}\left(\sum_{m \in \mathbb{Z}}\left|c_{k-m}\right| \sup _{|y| \leq \delta}\left|\varphi_{N}(x+y+m)-\varphi_{N}(x+m)\right|\right)^{2} d x .
\end{gathered}
$$

By induction method and properties of $\varphi_{N}$, we can easily check that

$$
\sum_{k \in \mathbb{Z}} \sup _{x \in[0,1]}\left|\varphi_{N}^{\prime}(x+k)\right| \leq N
$$

From $(\triangle)$ and properties of $\varphi_{N}$, we can obtain the following estimate:

$$
\begin{gathered}
\left\|o s c_{\delta}(f)\right\|_{2}^{2} \leq \int_{0}^{1} \sum_{k \in \mathbb{Z}}\left(\sum_{m \in \mathbb{Z}}\left|c_{k-m}\right|^{2} \sup _{|y| \leq \delta}\left|\varphi_{N}(x+y+m)-\varphi_{N}(x+m)\right|^{2}\right) d x \\
\leq \sum_{k \in \mathbb{Z}}\left|c_{k}\right|^{2} \int_{0}^{1}\left(\sum_{m \in \mathbb{Z}} \sup _{|y| \leq \delta}\left|\varphi_{N}(x+y+m)-\varphi_{N}(x+m)\right|\right)^{2} d x
\end{gathered}
$$




$$
\begin{gathered}
\leq \delta^{2} \sum_{k \in \mathbb{Z}}\left|c_{k}\right|^{2} \int_{0}^{1}\left(\sum_{m \in \mathbb{Z}} \sup _{|y| \leq \delta}\left|\varphi_{N}^{\prime}(x+\theta y+m)\right|\right)^{2} d x \\
\leq \delta^{2} \sum_{k \in \mathbb{Z}}\left|c_{k}\right|^{2} \int_{0}^{1}\left(\sum_{m \in \mathbb{Z}} \sup _{t \in[-1,2]}\left|\varphi_{N}^{\prime}(t+m)\right|\right)^{2} d x \\
\leq(3 N \delta)^{2} \sum_{k \in \mathbb{Z}}\left|c_{k}\right|^{2} .
\end{gathered}
$$

The last inequality of the above inequalities derives from $(\Delta)$. From $x \in$ $[0,1], 0<\theta<1$ and $0<\delta<1$, we have the forth inequality of the above inequalities.

Lemma 2.3 ${ }^{[5]}$ For any $f \in V^{p}(\varphi)$ the following conclusions hold:

1. $\left\|\operatorname{osc}_{\delta}(f)\right\|_{W\left(L^{p}\right)} \leq\|c\|_{\ell^{p}}\left\|\operatorname{osc}_{\delta}(\varphi)\right\|_{W\left(L^{1}\right)}$,

$2 .\left\|\sum_{k \in \mathbb{Z}} c_{k} \varphi(\cdot-k)\right\|_{W\left(L^{p}\right)} \leq\|c\|_{\ell^{p}}\|\varphi\|_{W\left(L^{1}\right)}$.

Lemma 2.4 If $X=\left\{x_{n}\right\}$ is real sequence with $\sup _{i}\left(x_{i+1}-x_{i}\right)=\delta<1$, then for any $f=\sum_{k \in \mathbb{Z}} c_{k} \varphi_{N}(\cdot-k) \in V_{N}$ we have $\|Q f\|_{L^{2}} \leq\|Q f\|_{W\left(L^{2}\right)} \leq$ $(3+\delta)\|c\|_{\ell^{2}}\|\varphi\|_{W\left(L^{1}\right)}$.

Proof. : For $f=\sum_{k \in \mathbb{Z}} c_{k} \varphi_{N}(\cdot-k)$ we have

$$
\begin{gathered}
|f(x)-(Q f)(x)|=\left|\sum_{j \in \mathbb{Z}}\left(f(x)-f\left(x_{j}\right)\right) \chi_{j, 1}(x)+\sum_{j \in \mathbb{Z}}\left(f(x)-f\left(x_{j+1}\right)\right) \chi_{j, 2}(x)\right| \\
\leq \sum_{j \in \mathbb{Z}}\left|f(x)-f\left(x_{j}\right)\right| \chi_{j, 1}(x)+\sum_{j \in \mathbb{Z}}\left|f(x)-f\left(x_{j+1}\right)\right| \chi_{j, 2}(x) \\
\leq \sum_{j \in \mathbb{Z}} \operatorname{osc}_{\frac{\delta}{2}}(f)(x) \chi_{j, 1}(x)+\sum_{j \in \mathbb{Z}} \operatorname{osc}_{\frac{\delta}{2}}(f)(x) \chi_{j, 2}(x) \\
=\operatorname{osc}_{\frac{\delta}{2}}(f)(x) .
\end{gathered}
$$

From this pointwise estimate and Lemma 2.3 we get

$$
\begin{gathered}
\|f-Q f\|_{W\left(L^{2}\right)} \leq\left\|o s c_{\frac{\delta}{2}}(f)\right\|_{W\left(L^{2}\right)} \\
\leq\|c\|_{\ell^{2}}\left\|O S c_{\frac{\delta}{2}}\left(\varphi_{N}\right)\right\|_{W\left(L^{1}\right)} .
\end{gathered}
$$

And by the results of [7] or [8] we know

$$
\left\|\operatorname{osc}_{\frac{\delta}{2}}\left(\varphi_{N}\right)\right\|_{W\left(L^{1}\right)} \leq(2+\delta)\left\|\varphi_{N}\right\|_{W\left(L^{1}\right)} .
$$

Putting the above discuss together, we have

$$
\begin{gathered}
\|Q f\|_{L^{2}} \leq\|Q f\|_{W\left(L^{2}\right)} \leq\|f-Q f\|_{W\left(L^{2}\right)}+\|f\|_{W\left(L^{2}\right)} \\
\leq(2+\delta)\|c\|_{\ell^{2}}\left\|\varphi_{N}\right\|_{W\left(L^{1}\right)}+\left\|\sum_{k \in \mathbb{Z}} c_{k} \varphi_{N}(\cdot-k)\right\|_{W\left(L^{2}\right)} \\
\leq(2+\delta)\|c\|_{\ell^{2}}\left\|\varphi_{N}\right\|_{W\left(L^{1}\right)}+\|c\|_{\ell^{2}}\left\|\varphi_{N}\right\|_{W\left(L^{1}\right)} \\
\leq(3+\delta)\|c\|_{\ell^{2}}\left\|\varphi_{N}\right\|_{W\left(L^{1}\right)} .
\end{gathered}
$$


The following Theorem 2.1 is one of our main theorems in this paper.

Theorem 2.1 Let $P$ be an orthogonal projection from $L^{2}(\mathbb{R})$ to $V_{N}$ and $Q[f(x)]=\sum_{j} f\left(x_{j}\right) \chi_{j, 1}(x)+\sum_{j} f\left(x_{j+1}\right) \chi_{j, 2}(x)$. If sampling set $X=\left\{x_{n}\right\}$ is a real sequence with $\sup _{i}\left(x_{i+1}-x_{i}\right)=\delta<1$ and $\frac{3 N \delta}{2 \sqrt{\sum_{k}\left|\hat{\varphi}_{N}(\pi+2 k \pi)\right|^{2}}}<1$, then any $f \in V_{N}$ can be recovered from its samples $\left\{f\left(x_{j}\right): x_{j} \in X\right\}$ on sampling set $X$ by the iterative algorithm

$$
\left\{\begin{array}{l}
f_{1}=P Q f \\
f_{n+1}=P Q\left(f-f_{n}\right)+f_{n}
\end{array}\right.
$$

The convergence is geometric, that is,

$$
\left\|f_{n+1}-f\right\|_{L^{2}} \leq\left(\frac{3 N \delta}{2 \sqrt{\sum_{k}\left|\hat{\varphi}_{N}(\pi+2 k \pi)\right|^{2}}}\right)^{n}\left\|f_{1}-f\right\|_{L^{2}}
$$

Proof. By $P f=f$ and $\|P\|_{o p}=1$, for any $f=\sum_{k \in \mathbb{Z}} c_{k} \varphi_{N}(\cdot-k) \in V_{N}$ we have

$$
\begin{gathered}
\|(I-P Q) f\|_{L^{2}}^{2}=\|P f-P Q f\|_{L^{2}}^{2} \leq\|P\|_{o p}^{2}\|f-Q f\|_{L^{2}}^{2}=\|f-Q f\|_{L^{2}}^{2} \\
=\int \mid \sum_{j \in \mathbb{Z}} f(x) \chi_{j, 1}(x)+\sum_{j \in \mathbb{Z}} f(x) \chi_{j, 2}(x)-\sum_{j \in \mathbb{Z}} f\left(x_{j}\right) \chi_{j, 1}(x) \\
-\left.\sum_{j \in \mathbb{Z}} f\left(x_{j+1}\right) \chi_{j, 2}(x)\right|^{2} d x \\
\leq \int\left(\sum_{j \in \mathbb{Z}}\left|f(x)-f\left(x_{j}\right)\right| \chi_{j, 1}(x)+\sum_{j \in \mathbb{Z}}\left|f(x)-f\left(x_{j+1}\right)\right| \chi_{j, 2}(x)\right)^{2} d x \\
=\int \sum_{j \in \mathbb{Z}}\left|f(x)-f\left(x_{j}\right)\right|^{2} \chi_{j, 1}(x)+\sum_{j \in \mathbb{Z}}\left|f(x)-f\left(x_{j+1}\right)\right|^{2} \chi_{j, 2}(x) d x \\
\leq \int \sum_{j \in \mathbb{Z}}\left|o s c_{\frac{\delta}{2}}(f)(x)\right|^{2} \chi_{j, 1}(x)+\sum_{j \in \mathbb{Z}}\left|o s c_{\frac{\delta}{2}}(f)(x)\right|^{2} \chi_{j, 2}(x) d x \\
=\left\|o s c_{\frac{\delta}{2}}(f)\right\|_{L^{2}}^{2} \leq\left(3 N \frac{\delta}{2}\right)^{2} \sum_{k \in \mathbb{Z}}\left|c_{k}\right|^{2}=\left(3 N \frac{\delta}{2}\right)^{2}\|c\|_{\ell^{2}}^{2} \\
\leq\left(\frac{3 N \delta}{2 \sqrt{\sum_{k}\left|\hat{\varphi}_{N}(\pi+2 k \pi)\right|^{2}}}\right)^{2}\|f\|_{L^{2}}^{2} .
\end{gathered}
$$

The third equality is from property $\sum_{j}\left(\chi_{j, 1}(x)+\chi_{j, 2}(x)\right)=1$. The forth equality derives from property $\operatorname{supp}_{j, i} \cap \operatorname{supp}_{k, l}=\emptyset(j \neq k, i \neq l)$. By Lemma 2.2 , the forth inequality holds. And we have

$$
\left\|f_{n+1}-f\right\|_{L^{2}}=\left\|f_{n}+P Q\left(f-f_{n}\right)-f\right\|_{L^{2}}=\left\|P Q\left(f-f_{n}\right)-\left(f-f_{n}\right)\right\|_{L^{2}}
$$




$$
\leq\|I-P Q\|\left\|f-f_{n}\right\|_{L^{2}} \leq \cdots \leq\|I-P Q\|^{n}\left\|f-f_{1}\right\|_{L^{2}} .
$$

Combining with the estimate of $\|I-P Q\|$, we can imply

$$
\left\|f_{n+1}-f\right\|_{L^{2}} \leq\left(\frac{3 N \delta}{2 \sqrt{\sum_{k}\left|\hat{\varphi}_{N}(\pi+2 k \pi)\right|^{2}}}\right)^{n}\left\|f_{1}-f\right\|_{L^{2}}
$$

Taking assumption $\frac{3 N \delta}{2 \sqrt{\sum_{k}\left|\hat{\varphi}_{N}(\pi+2 k \pi)\right|^{2}}}<1$, we know the algorithm is convergent.

In the following, we will show improved A-P iterative algorithm from weighted samples.

Theorem 2.2 Let $P$ be an orthogonal projection from $L^{2}(\mathbb{R})$ to $V_{N}$ and weight function satisfy the following three conditions (i)-(iii):

(i) $\operatorname{supp} \varphi_{x_{j}} \subset\left[x_{j}-\frac{a}{2}, x_{j}+\frac{a}{2}\right]$

(ii) there exist $M>0$ such that $\int\left|\varphi_{x_{j}}(x)\right| d x \leq M$,

(iii) $\int \varphi_{x_{j}}(x) d x=1$.

Let $A f(x)=\sum_{j}\left\langle f, \varphi_{x_{j}}\right\rangle \chi_{j, 1}(x)+\sum\left\langle f, \varphi_{x_{j+1}}\right\rangle \chi_{j, 2}(x)$. If sampling set $X=\left\{x_{n}\right\}$ is a real sequence with $0<\sup _{i}\left(x_{i+1}-x_{i}\right)=\delta<1$ and we choice proper $\delta$ and a such that $\frac{3 N}{\sqrt[2]{\sum_{k}\left|\hat{\varphi}_{N}(\pi+2 k \pi)\right|^{2}}}(\delta+a(3+a) M)<1$, then any $f \in V_{N}$ can be recovered from its weighted samples $\left\{\left\langle f, \varphi_{x_{j}}\right\rangle: x_{j} \in X\right\}$ on sampling set $X$ by the iterative algorithm

$$
\left\{\begin{array}{l}
f_{1}=P A f \\
f_{n+1}=P A\left(f-f_{n}\right)+f_{n}
\end{array}\right.
$$

The convergence is geometric, that is,

$$
\left\|f_{n+1}-f\right\|_{L^{2}} \leq\left(\frac{3 N \delta}{2 \sqrt{\sum_{k}\left|\hat{\varphi}_{N}(\pi+2 k \pi)\right|^{2}}}(\delta+a(3+a) M)\right)^{n}\left\|f_{1}-f\right\|_{L^{2}} .
$$

Proof. By $P f=f$ and $\|P\|_{o p}=1$, for any $f=\sum_{k \in \mathbb{Z}} c_{k} \varphi_{N}(\cdot-k) \in V_{N}$ we have

$$
\begin{gathered}
\|f-P A f\|_{L^{2}}=\|f-P Q f+P Q f-P A f\|_{L^{2}} \\
\leq\|f-Q f\|_{L^{2}}+\|Q f-A f\|_{L^{2}}
\end{gathered}
$$

From the proof of Theorem 3.1, we have the following estimate for $\|f-Q f\|_{L^{2}}$ :

$$
\|f-Q f\|_{L^{2}} \leq\left(\frac{3 N \delta}{2 \sqrt{\sum_{k}\left|\hat{\varphi}_{N}(\pi+2 k \pi)\right|^{2}}}\right)\|f\|_{L^{2}}
$$

For the second term $\|Q f-A f\|_{L^{2}}$ of (1) we have the pointwise estimate

$$
|(Q f-A f)(x)|
$$




$$
\begin{gathered}
=\left|\sum_{j}\left(f\left(x_{j}\right)-\left\langle f, \varphi_{x_{j}}\right\rangle\right) \chi_{j, 1}(x)+\sum_{j}\left(f\left(x_{j+1}\right)-\left\langle f, \varphi_{x_{j+1}}\right\rangle\right) \chi_{j, 2}(x)\right| \\
=\mid \int \sum_{j}\left(f\left(x_{j}\right)-f(\xi)\right) \varphi_{x_{j}}(\xi) \chi_{j, 1}(x) \\
+\sum_{j}\left(f\left(x_{j+1}\right)-f(\xi)\right) \varphi_{x_{j+1}}(\xi) \chi_{j, 2}(x) d \xi \mid \\
\leq M\left(\sum_{j} \operatorname{osc}_{\frac{a}{2}}(f)\left(x_{j}\right) \chi_{j, 1}(x)+\sum_{j} \operatorname{osc}_{\frac{a}{2}}(f)\left(x_{j+1}\right) \chi_{j, 2}(x)\right) \\
=M Q\left(\sum_{k \in \mathbb{Z}}\left|c_{k}\right| o s c_{\frac{a}{2}}\left(\varphi_{N}\right)(x-k)\right) .
\end{gathered}
$$

The above second equality derives from $\int \varphi_{x_{j}}(x) d x=1$. By $\int\left|\varphi_{x_{j}}(x)\right| d x \leq M$ and $\operatorname{supp} \varphi_{x_{j}} \subset\left[x_{j}-\frac{a}{2}, x_{j}+\frac{a}{2}\right]$, we know the above first inequality.

From this pointwise estimate and Lemma 2.4, it follows that:

$$
\begin{gathered}
\|Q f-A f\|_{L^{2}} \leq M(3+a)\|c\|_{\ell^{2}}\left\|\operatorname{osc}_{\frac{a}{2}}\left(\varphi_{N}\right)\right\|_{W\left(L^{1}\right)} \\
\leq M(3+a) \frac{\left\|\operatorname{osc}_{\frac{a}{2}}\left(\varphi_{N}\right)\right\|_{W\left(L^{1}\right)}}{\sqrt{\sum_{k}\left|\hat{\varphi}_{N}(\pi+2 k \pi)\right|^{2}}}\|f\|_{L^{2}} \\
\leq M(3+a) \frac{3 N a}{2 \sqrt{\sum_{k}\left|\hat{\varphi}_{N}(\pi+2 k \pi)\right|^{2}}}\|f\|_{L^{2}}
\end{gathered}
$$

By combining (1),(2) and (3), we can obtain

$$
\|I-P A\|_{L^{2}} \leq \frac{3 N}{2 \sqrt{\sum_{k}\left|\hat{\varphi}_{N}(\pi+2 k \pi)\right|^{2}}}(\delta+a(3+a) M) .
$$

Similar to the procedure in the proof of Theorem 3.1, we have

$$
\left\|f_{n+1}-f\right\|_{L^{2}} \leq\left(\frac{3 N}{2 \sqrt{\sum_{k}\left|\hat{\varphi}_{N}(\pi+2 k \pi)\right|^{2}}}(\delta+a(3+a) M)\right)^{n}\left\|f_{1}-f\right\|_{L^{2}} .
$$

Remark 1. Term $\left(\frac{1}{2}\right)^{n}$ is added in the expression of convergence rate. This improves the velocity of convergence. From the construction of operator $Q$ and $A$, we know why it appears in the expression of convergence rate.

The reconstruction algorithm in Theorem 2.1 and 2.2 require the existence of orthogonal projection from $L^{2}$ onto $V_{N}$. For this purpose, the following Theorem 2.3 will construct the orthogonal projection. We can find the similar proof of Theorem 2.3 in $[5,10]$. 
Theorem 2.3 Let $X=\left\{x_{n}\right\}$ be a real sequence such that $0<\sup _{i}\left(x_{i+1}-\right.$ $\left.x_{i}\right)=\delta<1$. Then

$$
P f=\sum_{x_{j} \in X}\left\langle f, k_{x_{j}}\right\rangle \tilde{k}_{x_{j}}
$$

is orthogonal projection from $L^{2}$ onto $V_{N}$, where $\left\{k_{x_{j}}\right\}$ and $\left\{\tilde{k}_{x_{j}}\right\}$.

Remark 2. : The above improved A-P iterative algorithm maybe be generalized to the case of $L^{p}(\mathbb{R})$ and $V^{p}(\varphi)$ whenever generator $\varphi$ belongs to $W_{0}\left(L^{1}\right)$. We will study it in future work.

\section{Conclusion}

In this paper we pay main attention on the weighted sampling and reconstruction in spline subspaces. We give some reconstruction methods from different weighted sampling in spline subspaces. The improved A-P iterative algorithm performs better than the old A-P algorithm. And we obtain the explicit convergence rate of the improved A-P iterative algorithm in spline subspaces.

\section{References}

1. Aldroubi, A., Gröchenig, K.: Beurling-Landau-type theorems for non-uniform sampling in shift invariant spline spaces. J. Fourier. Anal. Appl, 6(1)(2000) 93-103.

2. Sun, W. C., Zhou, X. W.: Average sampling in spline subspaces. Appl. Math. Letter, 15(2002)233-237.

3. Wang, J.: Spline wavelets in numerical resolution of partial differential equations, International Conference on Wavelet Analysis and its application, AMS/IP Studies in Advanced Mathematics, Vol 25(2002)257-277.

4. Chen, W., Itoh, S., Shiki, J.: On sampling in shift invariant spaces. IEEE Trans. Information. Theory 48(10)(2002)2802-2810.

5. Aldroubi, A., Gröchenig, K.: Non-uniform sampling and reconstruction in shiftinvariant spaces. SIAM Rev 43(4)(2001)585-620.

6. Chui, C. K.: An introduction to Wavelet, Academic Press, New York,1992.

7. Aldroubi, A.: Non-uniform weighted average sampling and reconstruction in shiftinvariant and wavelet spaces. Appl. Comput. Harmon. Anal 13(2002)156-161.

8. Aldroubi, A., Feichtinger,H.: Exact iterative reconstruction algorithm for multivate irregular sampled functions in spline-like spaces: The $L_{p}$ theory. Proc. Amer. Math. Soc 126(9)(1998)2677-2686.

9. Xian, J., Lin, W.: Sampling and reconstruction in time-warped spaces and their applications. to appear in Appl. Math. Comput, 2004.

10. Xian, J., Qiang, X. F.: Non-uniform sampling and reconstruction in weighted multiply generated shift-invariant spaces. Far. East. J. Math. Sci 8(3)(2003)281-293

11. Goh, S. S., Ong, I. G. H.: Reconstruction of bandlimited signals from irregular samples. Signal. Processing 46(3)(1995)315-329. 